\title{
Regenerative Medicine for the Aging Brain
}

\author{
Micaela Lopez-Leon, Paula C. Reggiani, Claudia B. Herenu, Rodolfo G. Goya* \\ Institute for Biochemical Research (INIBIOLP)-Cathedra of Histology B, Cathedra of Pathology B, School of Medicine, \\ National University of La Plata, Argentina.
}

Abstract

In the central nervous system, cholinergic and dopaminergic (DA) neurons are among the cells most susceptible to the deleterious effects of age. Thus, the basal forebrain cholinergic system is known to undergo moderate neurodegenerative changes during normal aging as well as severe atrophy in Alzheimer's disease (AD). Parkinson's disease (PD), a degeneration of nigro-striatal DA neurons is the most conspicuous reflection of the vulnerability of DA neurons to age. In this context, cell reprogramming offers novel therapeutic possibilities for the treatment of these devastating diseases. In effect, the generation of induced pluripotent stem cells (iPSCs) from somatic cells demonstrated that adult mammalian cells can be reprogrammed to a pluripotent state by the overexpression of a few embryonic transcription factors (TF). This discovery fundamentally widened the research horizon in the fields of disease modeling and regenerative medicine. Although it is possible to re-differentiate iPSCs to specific somatic cell types, the tumorigenic potential of contaminating iPSCs that failed to differentiate, increases the risk for clinical application of somatic cells generated by this procedure. Therefore, reprogramming approaches that bypass the pluripotent stem cell state are being explored. A method called lineage reprogramming has been recently documented. It consists of the direct conversion of one adult cell type into another by transgenic expression of multiple lineage-specific TF or microRNAs. Another approach, termed direct reprogramming, features several advantages such as the use of universal TF system and the ability to generate a rejuvenated multipotent progenitor cell population, able to differentiate into specific cell types in response to a specific differentiation factors. These novel approaches offer a new promise for the treatment of pathologies associated with the loss of specific cell types as for instance, nigral DA neurons (in PD) or basal forebrain cholinergic neurons in the early stages of AD. The above topics are reviewed here.

Keywords

brain aging; neurodegeneration; regenerative medicine; cell reprogramming; direct reprogramming; iPSCs; transdifferentiation; Parkinson; Alzheimer

'Corresponding author: Rodolfo G. Goya, INIBIOLP Faculty of Medicine, UNLP, CC 4551900 La Plata Argentina Tel: (54-221) 425-6735; E-mail:goya@isis.unlp.ar

Received Date: $25^{\text {th }}$ August-14

Accepted Date: $25^{\text {th }}$ September 2014

Published Date: $28^{\text {th }}$ September 2014

Regenerative Medicine and Cell Reprogramming

\section{Introductory Remarks}

The generation of induced pluripotent stem cells (iPSCs) from somatic cells has demonstrated that adult mammalian cells can be reprogrammed to a pluripotent state by the overexpression of a limited number of embryonic transcription factors (TF) [1]. The discovery of induced pluripotency represents the synthesis of scientific principles and technologies that have been developed over the last six decades. In 2006, Takahashi and Yamanaka demonstrated that the transfer of the four pluripotency genes Oct4, Sox2, cMyc and $\mathrm{Klf} 4$, to mouse fibroblasts can reprogram them, taking the cells to a stage in which they behave as embryonic stem cells (ESCs), which they termed iPSCs [1]. A year later, they demonstrated that the same genes, dubbed the Yamanaka genes, can reprogram human somatic cells to iPSCs [2].
Citation: López León M, Reggiani PC, Hereñú CB, Goya RG(2014)Regenerative Medicine for the Aging Brain. Enliven:J Stem Cells Regen Med 1(1):001

Copyright: @ 2014 Dr.Rodolfo G. Goya. This is an Open Access article published and distributed under the terms of the Creative Commons Attribution License, which permits unrestricted use, distribution and reproduction in any medium, provided the original author and source are credited. 
Cell reprogramming is an emerging technology that offers two major advantages over ESC-based therapeutic approaches. The first one is related to the fact that iPSCs can be generated from easily accessible somatic cells from the patient (skin fibroblasts, for instance). The iPSCs derived from the patient's own cells will be autologous for her/him and therefore will not induce immunologic rejection when implanted. This is a necessary requirement for implementing genuine personalized regenerative medicine. The second advantage is of ethical nature and stems from the fact that the use of iPSCs does not require the destruction of embryos as is the case for conventional ESC therapy.

Although it is possible to re-differentiate iPSCs into specific differentiated cell types by culturing such iPSCs in media supplemented with appropriate factors, the procedure as a whole is costly, arduous and lengthy. Since the protocols to generate iPSCs include a number of stages, the efficiency with which the final cell type is generated can be low. Furthermore, a number of concerns about the safety and fidelity of iPSC-derived cells need to be addressed before these cells can be used clinically [12]. It is important to note that one of the most commonly used assays for demonstrating pluripotency is the capacity to forming teratomas [13]. Therefore, the tumorigenic potential of contaminating iPSC that failed to differentiate, increases the risk for clinical application of somatic cells generated by this procedure [14]. Thus, other ways of reprogramming cells have been developed which involve direct conversion between cell types, thus avoiding passage through a pluripotency stage.
Transdifferentiation And Direct Reprogramming

Recently, a new cell reprogramming approach, known as lineage reprogramming or transdifferentiation, has emerged for the generation of specific cell types. It consists of the direct conversion of one adult cell type into another one by ectopic expression of multiple lineage-specific TF or microRNAs without the cell passing through the pluripotent SC state $[15,16]$. This strategy uses factors that show specific expression in target cells. Thus, the adenoviral-mediated gene transfer of a combination of three TF was reported to efficiently reprogram pancreatic exocrine cells into functional $\beta$ cells in mice, which constituted the first documented evidence of cell reprogramming in vivo by defined factors [17]. Recent studies have demonstrated that direct lineage reprogramming can yield a diverse range of medically relevant cell types, such as cardiomyocytes and neurons [1820]. Table 1 summarizes a number of lineage reprogramming protocols that have been documented for the generation of induced neurons. The directly reprogrammed cells exhibit equivalent functionality to the cells differentiated from iPSCs or their in vivo counterparts and show no tumorigenicity when transplanted in vivo [21,22]. However, cells generated by this procedure may demonstrate restricted proliferative capacity, limited cell type diversity and even senescence [23], which may in turn substantially compromise their potential application in regenerative therapy.

\begin{tabular}{|c|c|c|c|c|}
\hline Cell type of origin & Cell type induced & strategy & TFs used & Reference \\
\hline Embryonic stem cells & Induced DA neurons & Differentiation & Hes5, Nurr1 and Pitx3 & {$[35]$} \\
\hline $\begin{array}{c}\text { Mouse embryonic and postnatal } \\
\text { fibroblasts }\end{array}$ & iNs & $\begin{array}{l}\text { Lineage reprogramming } \\
\text { (transdifferentiation) }\end{array}$ & Ascl1, Brn2, Myt1l & [20] \\
\hline Mouse tail tip fibroblasts & Induced DA neurons & $\begin{array}{l}\text { Lineage reprogramming } \\
\text { (transdifferentiation) }\end{array}$ & $\begin{array}{l}\text { Acs11, Myt11, Brn2, Lmx1a, } \\
\text { Lmx1b, Nurr1, Pitx3 and EN1 }\end{array}$ & [19] \\
\hline Human fibroblasts & Induced DA neurons & $\begin{array}{l}\text { Lineage reprogramming } \\
\text { (transdifferentiation) }\end{array}$ & Ascl1, Brn2 and Myt1l & {$[36]$} \\
\hline $\begin{array}{l}\text { Fetal and postnatal human fibro- } \\
\text { blasts }\end{array}$ & iNs & $\begin{array}{l}\text { Lineage reprogramming } \\
\text { (transdifferentiation) }\end{array}$ & $\begin{array}{c}\text { Ascl1, Brn2, Myt1l and bHLH } \\
\text { NeuroD1 }\end{array}$ & [39] \\
\hline Mouse and human fibroblasts & Induced DA neurons & $\begin{array}{l}\text { Lineage reprogramming } \\
\text { (transdifferentiation) }\end{array}$ & Ascl1, Nurr1 and Lmx1a & {$[37]$} \\
\hline $\begin{array}{l}\text { Postnatal and adult human pri- } \\
\text { mary dermal fibroblasts }\end{array}$ & iNs & $\begin{array}{l}\text { Lineage reprogramming } \\
\text { (transdifferentiation) }\end{array}$ & miR-124, Myt1l and Brn2 & [40] \\
\hline Hepatocytes & iNs & $\begin{array}{l}\text { Lineage reprogramming } \\
\text { (transdifferentiation) }\end{array}$ & Ascl1, Brn2 and Mytl1 & {$[41]$} \\
\hline Human fibroblasts & DA neuron-like cells & $\begin{array}{l}\text { Lineage reprogramming } \\
\text { (transdifferentiation) }\end{array}$ & $\begin{array}{c}\text { Mash1, Ngn2, Sox2, nurr1 and } \\
\text { Pitx3 }\end{array}$ & [38] \\
\hline
\end{tabular}

Table 1.- Reprogramming Somatic Cells into Induced Neurons. DA: Dopaminergic; iNs: Induced Neuronal Cells. 
Another approach to regenerative medicine, termed direct reprogramming, is also emerging as an alternative to reprogramming via iPSC. The procedure uses fully differentiated somatic cells and converts them into other differentiated cell types, bypassing an intermediate pluripotent state. While in transdifferentiation, the conversion towards the cell lineage of interest is pushed by overexpression of lineage-specific factors, the pluripotency factor-mediated direct reprogramming (PDR) strategy [24] uses the same set of pluripotency factors as iPSC reprogramming, the Yamanaka genes, but they are expressed over a shorter period, usually 3 to 6 days. During this procedure, epigenetically unstable (EU) intermediate cells, which are responsive to differentiation factors, are generated. This new method has several advantages such as the use of a universal pluripotency gene system and the ability to generate a rejuvenated multipotent progenitor cel population, able to differentiate into various tissue-specific destination cells under specific conditions [24-26]. Table 2 lists specific reports of PDR.

Conventional lineage reprogramming and direct reprogramming share in common a direct conversion from one cellular type to another and are patientspecific. Both approaches avoid passing through an intermediate pluripotent stage, relying on specific signals to help the original cells reach the desired celltype destination. In contrast to lengthy iPSC-mediated cell type conversion, in the direct approaches the conversion usually occurs in a short period of time.

The Promise of Cell Reprogramming for the Aging Brain Brain Aging

Aging is associated with a progressive increase in the incidence of neurodegenerative diseases in both laboratory animals and humans. In the central nervous system (CNS), cholinergic and dopaminergic (DA) neurons are amongst the cells most susceptible to the deleterious effects of age and environmental insults. Thus, the basal forebrain cholinergic system is known to undergo moderate neurodegenerative changes during normal aging as well as severe atrophy in Alzheimer's Disease (AD). In fact, the cholinergic degeneration in $A D$ seems to occur against a background of age-related atrophy and the exacerbated atrophy in AD can be detected at very early stages of cognitive impairment [27]. In rats, aging is associated with degenerative and/or atrophic changes in the forebrain cholinergic system and these morphologic changes are paralleled by a decline in spatial learning ability [28].

Parkinson's disease (PD) is a neurological disorder characterized by the degeneration and progressive loss of DA neurons in the midbrain substantia nigra, leading to a reduction of dopamine in the striatum [29]. PD affects $0.1-0.3 \%$ of the population and is the most conspicuous reflection of the vulnerability of DA neurons to age. In rats, aging brings about a progressive degeneration and loss of another group of central DA neurons namely, the hypothalamic tuberoinfundibular dopaminergic (TIDA) neurons, which are involved in the tonic inhibitory control of prolactin secretion and lactotropic cell proliferation in the adenohypophysis [30]. Progressive dysfunction and loss of TIDA neurons during normal aging is associated in the female rat, with chronic hyperprolactinemia [31] and the development of pituitary prolactinomas [32]. Although aging rats do not develop parkinsonian symptoms, even at 32 months of age, they lose $35-40 \%$ nigral DA neurons and show a marked decline in motor performance [33].
In humans, normal aging is also associated with a decline in motor performance and a progressive loss of nigral DA neurons [34]. Therefore, progressive decline in cognitive function and central DA activity seems to represent basic features of normal aging both in humans and laboratory rodents. Exacerbation of these processes would lead to $A D$ and $P D$, respectively.

In this context, SC therapy and cell reprogramming emerge as powerful technologies that promise to make it possible implementing personalized regenerative medicine aimed at preventing or delaying the progress of $A D$ and PD. There is also a growing interest in the use of neural stem cells (NSCs) engineered to express oncolytic genes, for the treatment of brain tumors but the topic is beyond the scope of this article and will not be reviewed here.

\section{Reprogramming Somatic Cells to Induced Neurons}

There is a keen interest in obtaining mature neurons and neural precursors from somatic cells (e.g., fibroblasts) by transdifferentiation and direct reprogramming, which can later be used for implementing cell therapy for neurodegenerative pathologies like $A D$ and PD. It has been demonstrated that non-neural human somatic cells, as well as pluripotent SC, can be directly converted into neurons by lineage-determining TF. Currently, DA neurons can be obtained through differentiation from ESCs [35] and by direct conversion from fibroblasts $[19,36-38]$. It was initially shown that mouse embryonic and postnatal fibroblasts can be efficiently converted into functional neurons in vitro by transfer of the genes for only three neuronal lineage-specific TF namely, Ascl1, Brn2 and Myt11 [20]. The induced neuronal cells (iNs) express multiple neuron-specific proteins, generate action potentials and form functional synapses. When they are combined with the basic helix-loop-helix NeuroD1 factor, these three TF could also convert human fibroblasts into iNs that display typical neural morphologies and express multiple neural markers [39]. These human iNs are able to generate action potentials and to receive synaptic contacts with mouse cortical neurons in co-culture. Subsequently, it was reported that a combination of the TF Ascl1, Nurr1 and Lmxla was able to generate functional DA neurons from mouse and human fibroblasts without reverting to a progenitor cell stage [37]. This study also showed that DA neurons can be generated from cells of patients with PD. The combination of a microRNA (miR-124) and two TF (Myt1l and Brn2) is sufficient to directly reprogram postnatal and adult human primary dermal fibroblasts to functional neurons under precisely defined conditions [40]. These human iNs exhibit typical neural morphology and marker gene expression, fire action potentials and produce functional synapses between each other. These findings clearly show that the overexpression of a few 'master' factors is sufficient to drive relatively rapid and direct specific lineage changes in cells derived from different embryonic layers. In effect, lineage conversion is not restricted to within the same lineage or germ layer, since mesodermal source fibroblasts give rise to neurons, which are cells derived from the ectoderm. Additionally, it was demonstrated that terminally differentiated hepatocytes derived from the endoderm can be converted into iNs by overexpression of Ascl1, Brn2 and Mytl1 [41]. Additional studies have demonstrated that expression of subtype-specific regulatory factors in mouse and human fibroblasts results in the establishment of specific neuronal subtypes, like DA neurons [36-38]. 
Generation of Induced Neural Stem Cells and Neural

Progenitor Cells from Fibroblasts

Generation of iNs from non-neural lineages could have important implications for studies of neural development and neurological disease modeling because transdifferentiated neurons can be obtained from patients. Furthermore, they can be also used for producing neurons for regenerative medicine. However, terminally differentiated iNs are not adequate for transplantation [42] because, due to their limited ability to proliferate, only few cells usually survive and become functionally integrated to the brain [43]. Therefore, the treatment effectiveness of iNs transplantation is not optimal. Because differentiated cells are post-mitotic and non-dividing, the process of generating sufficient numbers of cells for further basic and clinical applications constitutes a significant challenge. Progenitors and precursors should be advantageous in handling and obtaining the cells in vitro as well as for proper integration in vivo.

In order to overcome the above hurdles, various studies have focused on the generation of induced NSCs (iNSCs) and neural progenitor cells (NPCs) from fibroblasts [25, 44-49]. These cells can differentiate into neurons and glial cells, the two major cell types in the CNS [50]. While NSCs are selfrenewing cells capable of producing neurons, astrocytes and oligodendrocytes [51], NPCs have limited self-renewal capacity. There are two main strategies to reprogram fibroblasts to iNSCs or NPCs: a direct method and an indirect method (PDR) that involves an intermediate destabilized state (see above).
Table 2 lists several reports using PDR or lineage reprogramming to obtain iNSCs and iNPCs. The PDR strategy was initially used by Kim et al. [25] who showed that transient induction of the four pluripotency factors (Oct4 Sox2, Klf4 and c-Myc) under the control of a DOX-inducible promoter for 3-6 days, followed by appropriate signaling inputs, can efficiently transdifferentiate fibroblasts into functional iNSC/NPCs. The fact that a number of studies have indicated that iPSCs are generated in a sequential and stochastic manner [52-54], led Kim et al. to hypothesize that they could be able to manipulate cells at an early and epigenetically highly unstable state induced by the Yamanaka reprogramming factors. Different conditions could potentially give rise to a multitude of cell types with more stable epigenetic profiles [55]. When such EU mouse fibroblasts are grown in a medium that contains the neurogenic molecules, fibroblast growth factor 2 (FGF2), FGF4 and epidermal growth factor (EGF), colonies of cells showing many of the features of NPCs are generated after 8-9 days in culture. This process is highly specific and efficient, requiring only a single step that is completed within 13 days, yielding almost $100 \%$ newly generated colonies that were mostly composed of NPCs [25]. Compared with iNs, NPCs have the distinct advantage of being expandable in vitro and retaining the ability to give rise to multiple neuronal subtypes and glial cells. Another advantage of this method is the use of general reprogramming TF instead of lineage-specific TF.

\begin{tabular}{|l|l|l|l|l|}
\hline Cell type of origin & Cell type induced & Strategy & TFs used & Reference \\
\hline Mouse fibroblasts & NSC/NPCs & $\begin{array}{l}\text { Direct reprogram- } \\
\text { ming strategy } \\
\text { (PDR) }\end{array}$ & $\begin{array}{l}\text { Oct4, Sox2, } \\
\text { Klf4, c-Myc }\end{array}$ & {$[25]$} \\
\hline Mouse fibroblasts & NSCs & PDR & $\begin{array}{l}\text { Sox2, KIf4 and } \\
\text { c-Myc }\end{array}$ & {$[48]$} \\
\hline Mouse and human fibroblasts & NSCs & PDR & Sox2 & {$[45]$} \\
\hline Mouse embryonic fibroblasts & Bipotent NPC/tripotent & $\begin{array}{l}\text { Lineage reprogram- } \\
\text { ming (transdifferen- } \\
\text { tiation) }\end{array}$ & $\begin{array}{l}\text { Sox2 and FoxG1/ } \\
\text { Sox2, FoxG1 and } \\
\text { Brn2/FoxG1 and } \\
\text { Brn2 }\end{array}$ & {$[56]$} \\
\hline Mouse fibroblasts & NSCs & $\begin{array}{l}\text { Lineage reprogram- } \\
\text { ming (transdifferen- } \\
\text { tiation) }\end{array}$ & $\begin{array}{l}\text { Sox2, c-Myc, KIf4 } \\
\text { and Brn4 }\end{array}$ & {$[57]$} \\
\hline
\end{tabular}

Table 2.- Generation of Induced Neural -Stem Cells and -Progenitor Cells from Fibroblasts. NSCs: neural stem cells, NPCs: neural progenitor cells 
Thier et al. [48] showed that constitutively inducing Sox2, Klf4 and c-Myc while strictly limiting Oct4 activity to the initial 5-day phase of reprogramming, generated iNSCs. These iNSCs that could be expanded for more than 50 passages, uniformly displayed morphological and molecular features of brain-derived NSCs and had a genome-wide transcriptional profile similar to them. Thus, self-renewable and multipotent iNSCs devoid of tumorigenic potential, can be generated directly from fibroblasts by restricted pluripotency gene overexpression. The generation of iNSCs from mouse and human fibroblasts by direct reprogramming with a single factor, Sox2, was also reported [45]. These iNSCs express NSC markers and resemble wild-type NSCs in their morphology, self-renewal ability to form neurospheres and gene expression profiles. They can differentiate into several types of mature neurons indicating multipotency. Implanted iNSCs can survive and integrate in mouse brains and, unlike iPSC-derived NSCs, they do not generate tumors. These iNSCs showed an extensive self-renewal capacity compared with the limited passaging ability of the iNSC/NPCs generated by Kim et al. [25], which could be expanded for only a few passages, thus excluding the possibility that the cells have a permanent self-renewing capacity, a critical requirement for clinical applicability. Besides, the cells obtained by Kim et al. apparently lacked the potential to differentiate into oligodendrocytes.

While it has been shown that defined sets of TF are sufficient to convert fibroblasts directly into iNs, for some applications it would be desirable to convert fibroblasts into proliferative NPCs instead of neurons. Lujan et al. [56] demonstrated that it is possible to obtain NPCs using the same main approach used for generating iNs, which is a strategy different from that of Kim et al. Towards this goal, the approach started with a set of 11 candidate genes for TF highly expressed in NPCs which were transferred to mouse embryonic fibroblasts by means of retroviral vectors. Using stepwise elimination, they found that Sox2 and FoxG1 are capable of generating clonal self-renewing, bipotent induced NPCs that gave rise to astrocytes and functional neurons. When the gene for Brn2 was added, tripotent NPCs were generated which could be differentiated not only into neurons and astrocytes but also into oligodendrocytes. Furthermore, FoxG1 and Brn2 alone were also capable of inducing NPC-like cells, which generated less mature neurons, although they did produce astrocytes and even oligodendrocytes capable of integration into dysmyelinated Shiverer mouse brains.
In a similar reprogramming strategy for the direct reprogramming of fibroblasts into iNSCs, Han et al. [57] started with the above list of 11 candidate genes for TF, three SC factors, Sox2, Klf4, and c-Myc, together with eight neural specific TF. They then tested different combinations and by systematic elimination, the list of effective factors was reduced to a smaller number in the reprogramming cocktail. They showed that Sox2, c-Myc, Klf4 and Brn4 were active factors sufficient to induce direct transdifferentiation of mouse fibroblasts into iNSCs, in what was a gradual process in which the fibroblast transcriptional program was silenced over time. These iNSCs exhibit cell morphology, gene expression, epigenetic features, differentiation potential and self-renewal capacity, as well as in vitro and in vivo functionality similar to that of wild-type NSCs.

The clinical applications of iNSCs and NPCs are significantly compromised by the fact that the reprogramming strategy to generate them involved integrative gene transfer with the well-known risk of insertional mutagenesis or gene silencing. We hypothesize that adenoviral vectors offer a safer reprogramming alternative and have thus, designed a regulatable helper-dependent (HD) adenovector expressing the four pluripotency genes Oct4, Sox2, KIf4 and c-Myc, as well as the humanized green fluorescent protein (hGFP) gene which should allow us to implement the strategy of Kim et al. [25] in a non integrative fashion (Figure. 1). In order to overcome the lower transduction efficiency of adenoviral as compared to retroviral vectors, we plan to use the magnetofection technique during cell reprogramming. Magnetofection is based on complexing adenovectors to magnetic nanoparticles which under the influence of a magnetic field, efficiently enter the target cells. This procedure markedly increased transduction efficiency of HD-adenovectors in cell culture and in vivo [58]. Using the adenovector shown in (Figure. 1) and the magnetofection technique, we plan to implement a direct reprogramming protocol on mouse and human fibroblasts in which the four Yamanaka pluripotency genes will be expressed for a short time (4 days) in order to obtain EU fibroblasts which are responsive to a variety of differentiation factors. These EU fibroblasts will then be exposed to suitable neurogenic factors in order to induce NPCs and terminally differentiated neurons.

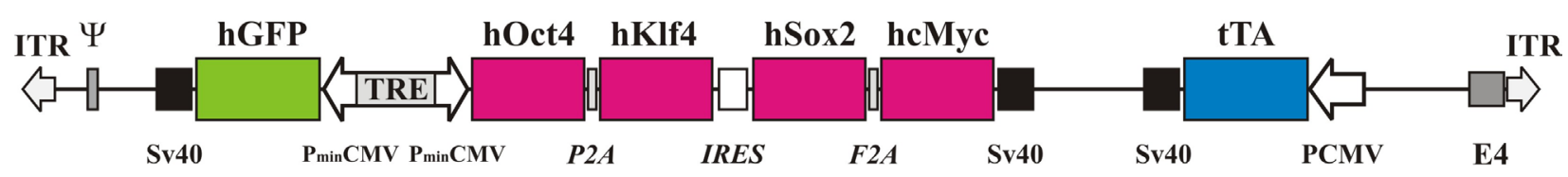

\section{HD-RAd-(GFP-TRE-STEMCCA)-(tTA)}

Figure 1. Diagrammatic Representation of the Genome of an Hd-Adenovector Suitable for Non Integrative Cell Reprogramming.

Under basal conditions the vector expresses the four Yamanaka genes and the gene for hGFP. Expression of pluripotency genes and hGFP can be readily inhibited by doxycycline addition to the culture medium. TRE, tetracycline-responsive element; PminCMV, human cytomegalovirus minimal promoter; PCMV, CMV full promoter; tTA, tetracycline-responsive transcriptional activator; hGFP, Reporter cDNA; SV4OpA, simian virus 40 polyadenylation signal; $\psi$, packaging signal; ITR, inverted terminal repeats; P2A, F2A, CHYSEL self-processing sequences; IRES, internal ribosome entry site; hOct4, hKIf4, hSox2, hcMyc, Yamanaka pluripotency genes. 
The Potential of Cell Reprogramming for the Treatment of PD and AD

The absence of efficacious therapies for neurological disorders has increased the interest in regenerative medicine based on cellular approaches, particularly SC technology. Stem cell therapy has been attempted in animal models and also in some clinical trials for neurodegenerative diseases, such as $P D, A D$, multiple sclerosis and amyotrophic lateral sclerosis [59-65] as well as in ischemic brain and spinal cord traumatic lesions [66, 44]. Different kinds of SC have been used for the treatment of neurological diseases [67] and, although the results of transplantation in animal models were encouraging, human clinical trials have not produced the same results.

\section{Therapeutic Potential of Cell Reprogramming for PD}

The use of ESCs as a source of DA neurons for the treatment of PD is a topic of growing interest and there is evidence that DA neurons derived from mouse ESCs survive for extended periods (over 37 weeks) in rodent models of PD [68]. Furthermore, mouse ESCs can spontaneously differentiate into DA neurons when implanted in the striatum of rats whose nigral DA neurons have been pharmacologically lesioned [69]. The newly generated DA neurons cause a gradual and sustained behavioral reversion of DA-mediated motor asymmetry. Like ESCs, iPSCs can be induced to differentiate into DA neurons and can be subsequently implanted into the brain of rat models of PD where they are able to improve behavior [63]. The possibility to induce NSCs from human somatic cells, e.g. fibroblasts, has opened new horizons for research in human disease modeling and cellular therapeutic applications in the neurological field. Cell reprogramming offers a major advantage over ESC-based therapeutic approaches for PD and AD. In effect, iPSCs as well as iNSCs and NPCs can be derived from easily accessible somatic cells from the patient (skin fibroblasts, for instance). The use of iNSCs may overcome some of the drawbacks of SC therapy. The fact that iNSCs can be expanded in vitro for a potentially unlimited number of passages, which is not the case of iNs, constitutes a convenient feature as clinical applications involving SC transplantation usually require large amounts of donor cells. As mentioned above, the induced neurons derived from the patient's own cells will be autologous for him/her and therefore will not induce immunologic rejection when implanted. This could reduce rejection problems that manifest during use of ESCs or other SC derived from different donors. Given that iNSCs are committed towards neural lineage, they are able to generate the three major neural cell types [45], enabling them to be adaptable with a lower risk of neoplastic formation $[14,70,71]$. In contrast, ESCs such as iPSCs are able to differentiate into a wide range of cell types, showing high plasticity and manifesting tumorigenic potential in transplanted animals $[14,72]$. Thus, iNSCs can differentiate into the same type of cells of their immediate environment, while still maintaining the ability to replicate after transplantation [46, 73], which is important for treating pathologies that are induced by both degeneration of brain parenchyma and deregulation of inflammatory cells, requiring replacement of both components $[44,74]$. It will also be of interest to explore the possibility of inducing transdifferentiation directly in the tissue for regeneration and repair in situ. In this way, it may be possible to use iNSCs to develop new advanced therapeutic approaches for neurodegenerative disorders.
However, there is evidence that NSC are more likely to differentiate into glial cells than into functional neurons after transplantation [75, 76], which is a disadvantage for neuron-replacement therapy of neurodegenerative diseases. As the PDR approach can generate proliferating iNSCs under appropriate environmental conditions, DA progenitors (DAP) also can be generated under appropriately modified environment conditions by the same strategy. Although these DAP are more committed than general NSCs they are not terminally differentiated neurons. Recently, Kim et al. [77] showed that mouse fibroblasts can be directly reprogrammed into midbrain-specific DAP through the transient expression of the four Yamanaka genes under DA neuron-specific and intermediate cell-enriching conditions. This study demonstrates the feasibility of inducing a direct cell fate alteration from fibroblast to specific neural progenitors through a PDR strategy and provides another novel route for obtaining useful progenitors for potential therapies and studies on various neural diseases.

\section{Therapeutic Potential of Cell Reprogramming for AD}

The initial stages of $A D$ are characterized by an early substantial loss of basal forebrain cholinergic neurons (BFCN) which leads to deficits in spatial learning and memory. Consequently, cholinergic neuron replacement constitutes a relevant therapeutic goal and, interestingly, it has been recently shown that BFCN can be consistently derived from human ESCs [78]. It has been also demonstrated that functional neurons can be generated by reprogramming skin fibroblasts from normal individuals and familiar $A D$ patients [79].

Neural restricted progenitors (NRPs), also known as neuroblasts, represent a type of transitional intermediate cells that lie between multipotent NPCs and terminally differentiated neurons during neurogenesis. These NRPs have the ability of self-renewal and migration in the nervous system [ 80 , 81]. They can differentiate into neurons rather than glial cells in vivo and in vitro [82, 83], which constitutes an important advantage for cellular therapy of human neurodegenerative diseases. When injected into the subventricular zone, NRPs can migrate extensively and integrate into different regions of the brain, to then differentiate into various subtypes of neurons, contributing to brain plasticity and repair [81]. Unfortunately, it is difficult to obtain highly purified NRPs from normal nervous tissue [80, 84], which prevents further studies and applications that require relatively large number of cells. Recently, it was demonstrated that by using only three defined factors, Sox2, c-Myc and either Brn2 or Brn4, human fetal fibroblasts can be converted to human induced NRPs (hiNRPs) [85]. These hiNRPs exhibit distinct neuronal characteristics including a capacity for self-renewal, expression of multiple neuronal markers, neuron-like morphology and a neuronal genome-wide transcriptional profile. They could be differentiated into various terminal neurons with functional membrane properties, but not into glial cells. Thus, the direct conversion of hiNRPs from somatic cells with a high efficiency may provide a new source of cells for neuronal development studies as well as for cellular replacement therapy of human degenerative diseases, such as PD, AD and Huntington's chorea. 
Concluding Remarks

The increase of the elderly population is an almost worldwide phenomenon. Consequently, the incidence of age-related neurological (and other) pathologies like $P D$ and $A D$ is becoming a problem of significant medical and economic impact which is further exacerbated by exposure of the general population to increasing levels of environmental pollutants. In this context, research and development of novel therapeutic approaches and molecular tools like regenerative medicine and regulatable polycistronic non integrative vectors expressing suitable TF respectively, may open new avenues for the treatment of these devastating neurological pathologies. In recent years, cell reprogramming has emerged as a powerful technology that promises to make it possible to implement personalized regenerative medicine for neurodegenerative diseases. It seems therefore plausible to hypothesize that in the not-too-distant future a mature cell reprogramming technology will provide effective means for curing and functionally restoring the aging brain.

\section{Conflict of Interest}

The authors confirm that they have no conflicts of interest.

\section{Acknowledgements}

The authors are indebted to Ms. Yolanda E. Sosa for editorial assistance. Part of the work from our laboratory reviewed here was supported by grant \#PICT11-1273 and PICT-13-1590 from the National Agency for the Promotion of Science and Technology to RGG and by grant PIP0597 from the Argentine Research Council (CONICET) to RGG. PCR, CBH and RGG are CONICET career researchers. MLL is a CONICET doctoral fellow

\section{Reference}

1 Takahashi K, Yamanaka S (2006) Induction of pluripotent stem cells from mouse embryonic and adult fibroblast cultures by defined factors. Cell 126: 663-676

2 Takahashi K, Tanabe K, Ohnuki M, Narita M, Ichisaka T, et al. (2007) Induction of pluripotent stem cells from adult human fibroblasts by defined factors. Cell 13: 861-872.

3 Bayart E, Cohen-Haguenauer O (2013) Technological overview of iPS Induction from human adult somatic cells. Curr Gene Ther 13: 73-92.

4 Nedelec S, Onteniente B, Peschanski M, Martinat C (2013) Geneticallymodified human pluripotent stem cells: new hopes for the understanding and the treatment of neurological diseases? Curr Gene Ther 13: 111-119.

5 Nakagami $\mathrm{H}$, Nakagawa $\mathrm{N}$, Takeya $\mathrm{Y}$, Kashigawi $\mathrm{K}$, Ishida $\mathrm{C}$, et al. (2006) Model of vasculogenesis from embryonic stem cells for vascular research and regenerative medicine. Hypertension 48: 112-119.

6 Brunt KR, Weisel RD, Li RK (2012) Stem cells and regenerative medicine - future perspectives. Can J Physiol Pharmacol 90: 327-335.

7 Atala A (2012) Regenerative medicine strategies. J Pediatr Surg 47: $17-28$.
8 Boiani M, Schöler HR (2005) Regulatory networks in embryo-derived pluriาpotent stem cells. Nat Rev Mol Cell Biol 6: 872-884.

9 Yamashita JK (2010) ES and iPS cell research for cardiovascular regeneration. Exp Cell Res 316: 2555-2559.

10 Bu L, Jiang X, Martin-Puig S, Caron L, Zhu S, et al. (2009) Human ISL1 heart progenitors generate diverse multipotent cardiovascular cell lineages. Nature 460: 113-117.

11 Gepstein L (2002) Derivation and potential applications of human embryonic stem cells. Circ Res 91: 866-876.

12 Okano H, Nakamura M, Yoshida K, Okada Y, Tsuji O, et al. (2013) Steps toward safe cell therapy using induced pluripotent stem cells. Circ Res 112: 523-533.

13 Patel M, Yang S (2010) Advances in reprogramming somatic cells to induced pluripotent stem cells. Stem Cell Rev 6: 367-380.

14 Miura K, Okada Y, Aoi T, Okada A, Takahashi K, et al. (2009) Variation in the safety of induced pluripotent stem cell lines. Nat Biotechnol 27 : 743-745.

15 leda M (2013) Direct reprogramming into desired cell types by defined factors. Keio J Med 62: 74-82

16 Sancho-Martinez I, Baek SH, Izpisua Belmonte JC (2012) Lineage conversion methodologies meet the reprogramming toolbox. Nat Cell Biol 14: 892-899.

17 Zhou Q, Brown J, Kanarek A, Rajagopal J, Melton DA (2008) In vivo reprogramming of adult pancreatic exocrine cells to beta-cells. Nature 455: $627-632$.

18 leda M, Fu DJ, Delgado-Olguin P, Vedantham V, Hayashi $Y$, et al. (2010) Direct reprogramming of fibroblasts into functional cardiomyocytes by defined factors. Cell 142: 375-386.

19 Kim J, Su S, Wang H, Cheng AW, Cassady JP, et al. (2011) Functional integration of dopaminergic neurons directly converted from mouse fibroblats. Cell Stem Cell 9: 413-419.

20 Vierbuchen T, Ostermeier A, Pang ZP, Kokubu Y, Südhof TC, et al. (2010) Direct conversion of fibroblasts to functional neurons by defined factors. Nature 463: 1035-1041.

21 Liu GH, Yi F, Suzuki K, Qu J, Izpisua Belmonte JC (2012) Induced neural stem cells: a new tool for studying neural development and neurological disorders. Cell Res 22: 1087-1091.

22 Matsui T, Takano M, Yoshida K, Ono S, Fujisaki C, et al. (2012) Neura stem cells directly differentiated from partially reprogrammed fibroblasts rapidly acquire gliogenic competency. Stem Cells 30: 1109-1119.

23 Margariti A, Winkler B, Karamariti E, Zampetaki A, Tsai T, et al. (2012) Direct reprogramming of fibroblasts into endothelial cells capable of angiogenesis and reen $\neg$ dothelialization in tissue-engineered vessels. Proc natl Acad Sci USA 109: 13793-13798.

24 Kim J, Ambasudhan R, Ding S (2012) Direct lineage reprogramming to neural cells. Curr Opin Neurobiol 22: 778-784.

25 Kim J, Efe JA, Zhu S, Talantova M, Yuan X, et al. (2011) Direct reprogramming of mouse fibroblasts to neural progenitors. Proc nat Acad Sci USA 108: 7838-7843.

26 Efe JA, Hilcove S, Kim J, Zhou H, Ouyang K, et al. (2011) Conversion of mouse fibroblasts into cardiomyocytes using a direct reprogramming strategy. Nat Cell Biol 13: 215-222. 
27 Grothe M, Heinsen H, Teipel SJ (2012) Atrophy of the Cholinergic Basal Forebrain Over the Adult Age Range and in Early Stages of Alzheimer's Disease. Biol Psychiatry 71: 805-813.

28 Fischer W, Chen KS, Gage FH, Björklund A (1992) Progressive decline in spatial learning and integrity of forebrain cholinergic neurons in rats during aging. Neurobiol Aging 13: 9-23.

29 Gaillard A, Jaber M (2011) Rewiring the brain with cell transplantation in Parkinson's disease. Trends Neurosci 34: 124-133.

30 Sarkar DK, Gottschall PE, Meites J (1982) Damage to hypothalamic dopaminergic neurons associated with development of prolactinsecreting pituitary tumors. Science 218: 684-6.

31 Goya RG, Lu JKH, Meites J (1990) Gonadal function in aging rats and its relation to pituitary and mammary pathology. Mech Age Devel 56: 77-88.

32 Cónsole G, Gómez Dumm CL, Brown OA, Ferese C, Goya RG (1997) Sexual dimorphism in the age changes of the pituitary lactotrophs in rats. Mech Age Devel 95: 157-66.

33 Sánchez HL, Silva LB, Portiansky EL, Herenu CB, Goya RG, et al. (2008) Dopaminergic mesencephalic systems and behavioral performance in very old rats. Neuroscience 154: 1598-606.

34 Rudow G, O' Brien R, Savonenko AV, Resnick SM, Zonderman AB, et al. (2008) Morphometry of the human substantia nigra in ageing and Parkinson's disease. Acta Neuropathol 115: 461-70.

35 Ganat YM, Calder EL, Kriks S, Nelander J, Tu EY, et al. (2012) Identification of embryonic stem cell-derived midbrain dopaminergic neurons for engraftment. J Clin Invest 122: 2928-2939.

36 Pfisterer U, Kirkeby A, Torper O, Wood J, Nelander J, et al. (2011) Direct conversion of human fibroblasts to dopaminergic neurons. Proc natl Acad Sci USA 108: 10343-10348.

37 Caiazzo M, Dell'Anno MT, Dvoretskova E, Lazarevic D, Taverna S, et al. (2011) Direct generation of functional dopaminergic neurons from mouse and human fibroblasts. Nature 476: 224-227.

38 Liu X, Li F, Stubblefield EA, Blanchard B, Richards TL, et al. (2012) Direct reprogramming of human fibroblasts into dopaminergic neuronlike cells. Cell Res 22: 321-332.

39 Pang ZP, Yang N, Vierbuchen T, Ostermeier A, Fuentes DR, et al. (2011) Induction of human neuronal cells by defined transcription factors. Nature 476: 220-223.

40 Ambasudhan R, Talantova M, Coleman R, Yuan X, Zhu S, et al. (2011) Direct Reprogramming of Adult Human Fibroblasts to Functional Neurons under Defined Conditions. Cell Stem Cell 9: 113-118.

41 Marro S, Pang ZP, Yang N, Tsai MC, Qu K, et al. (2011) Direct lineage conversion of terminally differentiated heaptocytes to functional neurons. Cell Stem Cell 9: 374-382.

42 Rhee YH, Ko JY, Chang MY, Yi SH, Kim D, et al. (2011) Protein-based human iPS cells efficiently generate functional dopamine neurons and can treat a rat model of Parkinson disease. J Clin Invest 121: 23262335.

43 Zou Q, Yan Q, Zhong J, Wang K, Sun H, et al. (2014) Direct conversion of human fibroblasts into neuronal restricted progenitors. $\mathrm{J}$ Biol Chem 289: 5250-5260.

44 Wang L, Wang L, Huang W, Su H, Xue Y, et al. (2013) Generation of integration-free neural progenitor cells from cells in human urine. Nat Meth 10: 84-89.

45 Ring KL, Tong LM, Balestra ME, Javier R, Andrews-Zwilling $Y$, et al. (2012) Direct reprogramming of mouse and human fibroblasts into multipotent neural stem cells with a single factor. Cell Stem Cell 11: 100-109.

46 Lujan E, Chanda S, Ahlenius H, Sudhof TC, Wernig M (2012) Direct conversion of mouse fibroblasts to self-renewing, tripotent neural precursor cells. Proc natl Acad Sci USA 10: 2527-2532.
47 Han DW, Tapia N, Hermann A, Hemmer K, Hoing S, et al. (2012) Direct reprogramming of fibroblasts into neural stem cells by defined factors. Cell Stem Cell 10: 465-472.

48 Thier M, Worsdorfer P, Lakes YB, Gorris R, Herms S, et al. (2012) Direct conversion of fibroblasts into stably expandable neural stem cells. Cell Stem Cell 10: 473-479.

49 Sheng C, Zheng Q, Wu J, Xu Z, Wang L, et al. (2012) Direct reprogramming of Sertoli cells into multipotent neural stem cells by defined factors. Cell Res 22: 208-218.

50 Reynolds BA, Weiss S (1992) Generation of neurons and astrocytes from isolated cells of the adult mammalian central nervous system. Science 255: 1707-1710.

51 Gage FH (2000) Mammalian neural stem cells. Science 287: 14331438.

52 Stadtfeld M, Maherali N, Breault DT, Hochedlinger K (2008) Defining molecular cornerstones during fibroblast to iPS cell reprogramming in mouse. Cell Stem Cell 2: 230-240.

53 Brambrink T, Foreman R, Welstead GG, Lengner CJ, Wernig M, et al. (2008) Sequential expression of pluripotency markers during direct reprogramming of mouse somatic cells. Cell Stem Cell 2: 151-159.

54 Hanna J, Saha K, Pando B, van Zon J, Lengner CJ, et al. Direct cell reprogramming is a stochastic process amenable to acceleration. Nature 2009 462: 595-601.

55 Artyomov MN, Meissner A, Chakraborty AK (2010) A model for genetic and epigenetic regulatory networks identifies rare pathways for transcription factor induced pluripotency. PLOS Comput Biol 6: e1000785.

56 Lujan E, Chanda S, Ahlenius H, Südhof TC, Wernig M (2012) Direct conversion of mouse fibroblasts to self-renewing, tripotent neural precursor cells. Proc natl Acad Sci USA 109: 2527-2532.

57 Han DW, Tapia N, Hermann A, Hemmer K, Hoing S, et al. (2012) Direct reprogramming of fibroblasts into neural stem cells by defined factors. Cell Stem Cell 10: 465-472.

58 Schwerdt JI, Goya GF, Calatayud MP, Hereñú CB, Reggiani PC, et al. (2012) Magnetic field-assisted gene delivery: achievements and therapeutic potential. Curr Gene Ther 12: 116-126.

59 Karussis D, Petrou P, Kassis I (2013) Clinical experience with stem cells and other cell therapies in neurological diseases. J. Neurol Sci 324: 1-9.

60 Lee HJ, Lee JK, Lee H, Shin JW, Carter JE, et al. (2010) The therapeutic potential of human umbilical cord blood-derived mesenchymal stem cells in Alzheimer's disease. Neurosci Lett 481: 30-35.

61 Lunn JS, Sakowski SA, Federici T, Glass JD, Boulis NM, et al. (2011) Stem cell technology for the study and treatment of motor neuron diseases. Reg. Med 6: 201-213.

62 Moghadam FH, Alaie H, Karbalaie K, Tanhaei S, Nasr Esfahani MH, et al. (2009) Transplantation of primed or unprimed mouse embryonic stem cellderived neural precursor cells improves cognitive function in Alzheimerian rats. Differentiation 78: 59-68.

63 Politis M, Lindvall O (2012) Clinical application of stem cell therapy in Parkinson's disease. BMC Med 10: 1-7.

64 Wernig M, Zhao JP, Pruszak J, Hedlund E, Fu D, et al. (2008) Neurons derived from reprogrammed fibroblasts functionally integrate into the fetal brain and improve symptoms of rats with Parkinson's disease. Proc natl Acad Sci USA 105: 5856-5861.

65 Xuan AG, Luo M, Ji WD, Long DH (2009) Effects of engrafted neural stem cells in Alzheimer's disease rats. Neurosci Lett 450: 167-171.

66 Nandoe Tewarie RS, Hurtado A, Bartels RH, Grotenhuis A, Oudega M (2009) Stem cell-based therapies for spinal cord injury. J Spinal Cord Med 32: 105-114. 
67 Larijani B, Esfahani EN, Amini P, Nikbin B, Alimoghaddam K, et al. (2012) Stem cell therapy in treatment of different diseases. Acta Med Iran 50: 79-96.

68 Rodriguez-Gomez JA, Lu J-Q, Velasco I, Rivera S, Zaghbi SS, et al. (2007) Persistent dopamine functions of neurons derived from embryonic stem cells in a rodent model of Parkinson disease . Stem Cells 25: 918-928.

69 Bjorklund LM, Sanchez-Pernaute R, Chung S, Chen IY, Andersson T, et al. (2002) Embryonic stem cell develop into functional dopaminergic neurons after transplantation in a Parkinson rat model. Proc natl Acad Sci USA 99: 2344-2349.

70 Fong CY, Gauthaman K, Bongso A (2010) Teratomas from pluripotent stem cells: a clinical hurdle. J Cell Biochem 111: 769-781.

71 Yamanaka S (2009) A fresh look at iPS cells. Cell 137: 13-17.

72 Keirstead HS, Nistor G, Bernal G, Totoiu M, Cloutier F, et al. (2005) Human embryonic stem cell-derived oligodendrocyte progenitor cell transplants remyelinate and restore locomotion after spinal cord injury. J Neurosci 25: 4694-4705.

73 Son EY, Ichida JK, Wainger BJ, Toma JS, Rafuse VF, et al. (2011) Conversion of mouse and human fibroblasts into functional spinal motor neurons. Cell Stem Cell 9: 205-218.

74 Tsuji O, Miura K, Okada Y, Fujiyoshi K, Mukaino M, et al. (2010) Therapeutic potential of appropriately evaluated safe-induced pluripotent stem cells for spinal cord injury. Proc natl Acad Sci USA 107: 12704-12709.

75 Holmin S, Almqvist P, Lendahl U, Mathiesen T (1997) Adult nestinexpressing subependymal cells differentiate to astrocytes in response to brain injury. Eur J Neurosci 9: 65-75.

76 Johansson CB, Momma S, Clarke DL, Risling M, Lendahl U, et al. (1999) Identification of a neural stem cell in the adult mammalian central nervous system. Cell 96: 25-34.

77 Kim HS, Kim J, Jo Y, Jeon D, Cho YS (2014) Direct lineage reprogramming of mouse fibroblasts to functional midbrain dopaminergic neuronal progenitors. Stem Cell Res 12: 60-68.

78 Bissonnette CJ, Lyass L, Bhattacharyya BJ, Belmadani A, Miller RJ, et al. (2011) The controlled generation of functional basal forebrain cholinergic neurons from human embryonic stem cells. Stem Cells 29: 802-811.

79 Qiang L, Fujita R, Yamashita T, Angulo S, Rhinn H, et al. (2011) Directed conversion of Alzheimer's disease patient skin fibroblasts into functional neurons. Cell 146: 359-371.
80 Mayer-Proschel M, Kalyani AJ, Mujtaba T, Rao, MS (1997) Isolation of lineage-restricted neuronal precursors from multipotent neuroepithelial stem cells. Neuron 19: 773-785.

81 Yang H, Mujtaba T, Venkatraman G, Wu YY, Rao MS, et al. (2000) Region-specific differentiation of neural tube-derived neuronal restricted progenitor cells after heterotopic transplantation. Proc natl Acad Sci USA 97: 13366-13371.

82 Mo Z, Moore AR, Filipovic R, Ogawa Y, Kazuhiro I, et al. (2007) Human cortical neurons originate from radial glia and neuron-restricted progenitors. J Neurosci 27: 4132-4145.

83 Mujtaba T, Piper DR, Kalyani A, Groves AK, Lucero MT, et al. (1999) Lineage-restricted neural precursors can be isolated from both the mouse neural tube and cultured ES cells. Dev Biol 214: 113-127.

84 Kalyani AJ, Piper D, Mujtaba T, Lucero MT, Rao MS (1998) Spinal cord neuronal precursors generate multiple neuronal phenotypes in culture. J Neurosci 18: 7856-7868.

85 Zou Q, Yan Q, Zhong J, Wang K, Sun H, et al. (2014) Direct Conversion of Human Fibroblasts into Neuronal Restricted Progenitors. J Biol Chem 289: 5250-5260. 